\title{
Modelling and Analysing the Contract Net Protocol - Extension Using Coloured Petri Nets
}

\author{
Jonathan Billington, Amar Kumar Gupta, and Guy Edward Gallasch \\ Computer Systems Engineering Centre \\ University of South Australia \\ Mawson Lakes Campus, SA, 5095, Australia \\ \{jonathan.billington, amar.gupta, guy.gallasch\}@unisa.edu.au
}

\begin{abstract}
The Contract Net Protocol is a task allocation protocol that facilitates negotiation between bidders and an auctioneer in a MultiAgent System to form a contract. The extension allows the bidders to interact with more than one auctioneer concurrently, and to update their bids until a bid is granted. This introduces flexibility and ensures better selection of a bid. In this paper, we model the Contract Net Protocol extension with Coloured Petri Nets and show that it terminates correctly. We analyse the terminal states and prove that the agents have consistent beliefs at the end of the negotiations, and that there is no "dead code" in the procedures. Lastly, we show how the number of terminal states and channel bounds are related to the number of bidders.
\end{abstract}

Keywords: Contract Net Protocol - extension, Coloured Petri Nets, Verification.

\section{Introduction}

A Multi-Agent System 44 comprises a set of agents that interact with each other to achieve a goal. Typical agents constitute a service requesting agent, which requests a certain task to be performed, and a service providing agent, which performs the task. These agents undertake negotiations to form contracts. The Contract Net Protocol [7,19] is an elementary protocol that facilitates task allocation between an auctioneer (service requesting agent) and many bidders (service providing agents). This is extended to the Contract Net Protocol - extension [1] that allows the bidders to negotiate with multiple auctioneers simultaneously. This prevents the bidders from losing potential contracts with other auctioneers. Also, the protocol has two different phases of decision making; a provisional and a confirmed decision making phase. This feature allows bidders to submit updated bids and helps ensure that the auctioneers select the best bids. This work is motivated by researchers working on transport logistics [13.

In 3] we modelled the Contract Net Protocol [719] using Coloured Petri nets (CPNs) 910 and proved a number of properties. In doing so, we demonstrated the effectiveness of CPNs for this task, contrary to the claims made in the literature regarding the inadequacy of CPNs for modelling interaction protocols [112].

K. Suzuki et al. (Eds.): FORTE 2008, LNCS 5048, pp. 169 184, 2008.

(C) IFIP International Federation for Information Processing 2008 
In this paper, we extend our work to the Contract Net Protocol - extension (CNPext), represented using Protocol Flow Diagrams in [13. The protocol has iterative processes and the work presented in 1 proves that the protocol converges. The importance of the verification of properties of a protocol before implementation [2] has further stimulated this work. To the best of our knowledge, no work has been undertaken on the verification of the properties of this protocol.

This paper has a threefold contribution. Firstly, we present for the first time a model of CNP-ext. We analyse this model using state space techniques for any number of bidders up to 5 . Secondly, we show that the protocol terminates correctly and the agents have consistent beliefs regarding the contract at the end of negotiations. Finally, we conjecture relationships between the number of bidders and the number of terminal states and bounds on the underlying channel.

This paper is organised as follows. Section 2 provides an overview of the protocol and its operation. The CPN model of CNP-ext and its operation are presented in Section 3. Section 4 analyses the model and finally Section 5 presents conclusions and avenues for further work.

\section{Contract Net Protocol - Extension}

In contrast to the Contract Net Protocol that consists of a single auctioneer and multiple bidders, the Contract Net Protocol - extension (CNP-ext) 1 consists of multiple auctioneers negotiating with multiple bidders to form a contract. The auctioneers may negotiate with a number of bidders simultaneously. However, the auctioneers do not interact with each other and their negotiation processes are independent of each other. Similarly, the bidders may interact with multiple auctioneers at the same time, but not with each other. For simplicity, we describe below the CNP-ext in the context of a single auctioneer and multiple bidders.

The auctioneer initiates negotiation by sending a Task Announcement to the bidders, who respond with a Pre Bid (a temporary bid) for the task. When all the Pre Bids have been received the auctioneer selects the one it believes most suitable. This bid is provisionally granted and all others are provisionally rejected. The provisionally granted bidder then prepares and sends a Definitive Bid (a final bid) that may or may not be the same as the Pre Bid. All other bidders have the opportunity to update and re-submit a Pre Bid at this time.

When the auctioneer receives the Definitive Bid and all the updated Pre Bids from the provisionally rejected bidders, it compares the Definitive Bid to the rest of the updated Pre Bids. Two things could then happen. In the first scenario, if the Definitive Bid is still considered superior to all the updated Pre Bids, then the auctioneer sends a Confirm Grant to the corresponding bidder and a Confirm Reject to all other bidders. Negotiation would now be complete. In the second scenario, if an updated Pre Bid exists that is better than the Definitive Bid, then the auctioneer rejects (either Provisional Reject or Confirm Reject) the Definitive Bid. It then provisionally grants the new best Pre Bid and provisionally rejects the rest of the Pre Bids. The provisionally granted bidder then sends a Definitive Bid and the remaining bidders can again submit updated Pre Bids, and the process re- 


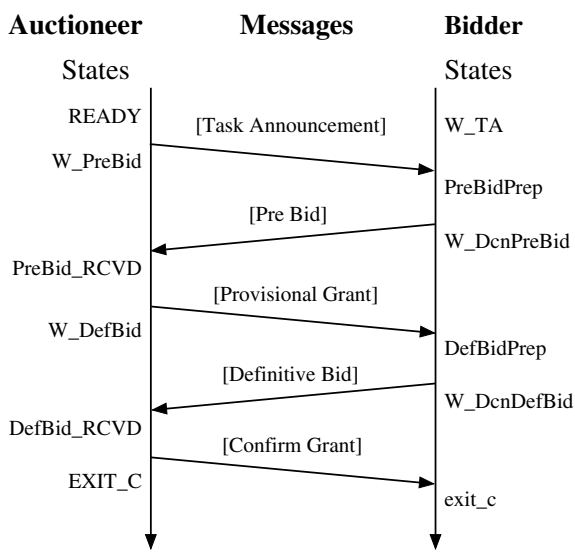

Fig. 1. Confirm Grant of a Definitive Bid

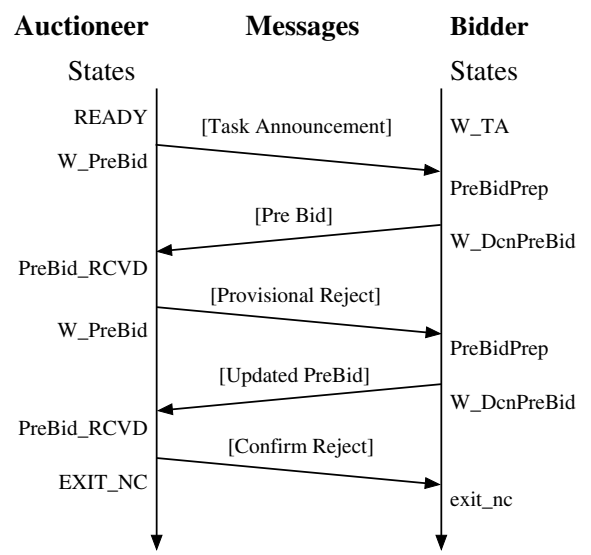

Fig. 2. Rejecting an Updated PreBid

peats iteratively. Negotiations will come to an end in one of two ways, either when the auctioneer confirms the grant of a Definitive Bid (and hence firmly rejects the remaining Pre Bids), or when there are no more updated Pre Bids to consider. The latter may arise if the auctioneer progressively terminates negotiations with each bidder that submits a Definitive Bid by sending a Confirm Reject in response, hence terminating negotiations with that bidder.

Multiple auctioneers may interact with the bidders simultaneously. When a Task Announcement is received from more than one auctioneer, a bidder will prioritise the tasks and send Pre Bids to any number of auctioneers. On receipt of a Provisional Grant in return, it prepares and sends a Definitive Bid, while a Provisional Reject causes the bidder to reprioritise its tasks and send updated Pre Bids. A Confirm Grant message commits the bidder to the task, while a Confirm Reject frees the bidder from any further negotiation with that auctioneer. The bidder could however continue negotiating with the other auctioneers.

Examples of interaction between an auctioneer and a single bidder are illustrated in the Time Sequence Diagrams (TSD) in Figs. 1 and 2. In each TSD the auctioneer is represented on the left and the bidder on the right. The changes in state for each are shown down each side. The states of the auctioneers and bidders are defined in Table1. There are seven possible states for each. READY and W_TA correspond to the initial states of the auctioneers and bidders respectively. The terminal states are EXIT_NC and EXIT_C (uppercase) for the auctioneers and exit_nc and exit_c (lowercase) for the bidders, following the convention of [13.

In Fig. 1, the auctioneer sends a Task Announcement to a bidder, which responds with a Pre Bid. On receipt of the Pre Bid, the auctioneer sends a Provisional Grant, which results in the bidder sending a Definitive Bid for the task. The auctioneer finally confirms this Definitive Bid to end the negotiation with a contract (auctioneer and bidder terminate in the EXIT_C and exit_c states respectively). Figure 2 shows a similar scenario, but this time the auctioneer provisionally rejects the Pre Bid. The bidder sends an Updated Pre Bid in re- 
Table 1. Representation of States

\begin{tabular}{|c|c|}
\hline Auctioneers & Bidders \\
\hline READY (READY to send a Task Announcement) & W-TA (Waiting for a Task Announcement) \\
W_PreBid (Waiting for Pre Bids) & PreBidPrep (Pre Bid Preparation) \\
PreBid_RCVD (Pre Bid ReCeiVeD) & W_DcnPreBid (Waiting for Decision on Pre Bid) \\
W_DefBid (Waiting for Definitive Bid) & DefBidPrep (Definitive Bid Preparation) \\
DefBid_RCVD (Definitive Bid ReCeiVeD) & W_DcnDefBid (Waiting for Decision on Definitive Bid) \\
EXIT_NC (EXIT with No Contract) & exit_nc (exit with no contract) \\
EXIT_C (EXIT with Contract) & exit_c (exit with contract) \\
\hline
\end{tabular}

sponse to the Provisional Reject, which the auctioneer finally rejects. Negotiation ends without a contract.

\section{CPN Model of the Contract Net Protocol - Extension}

Coloured Petri Nets are a form of High-level Petri net [8] in which tokens are arbitrarily complex data values and places are marked by multisets of such tokens. Each place is typed by a set of values, called a colour set, that specifies allowable values of tokens that can mark that place. In this section we firstly list the assumptions made when creating the model, define the data structures, variables and constants used to annotate the model, and finally describe the structure and operation of the model. We use the software tool, CPN Tools [10, to create and analyse the model. We assume basic familiarity with Petri net concepts, however for a detailed introduction to CPNs the reader is referred to [910].

\subsection{Assumptions}

When creating our CNP-ext model, we made the following assumptions:

1. All bidders are known to the auctioneers before the negotiations take place.

2. All messages (Task Announcement, Pre Bid, Definitive Bid, Provisional Grant, Provisional Reject, Confirm Grant and Confirm Reject) are represented by their name only, as other information contained in these messages does not affect the protocol's procedures.

3. All bidders have enough resources to bid, and will always bid, in response to all Task Announcements.

4. All the bids are received before the process of making a decision takes place, which means that we do not have to model a deadline.

5. The communication channel is lossless but reordering.

\subsection{Declarations}

Listing 1 shows the declarations for the CNP-ext CPN model. We describe the more significant declarations below. The identity of the auctioneers and bidders are represented by the colour sets AUC (line 3) and BDR (line 8) respectively. The identity of the auctioneers (AUC) and bidders (BDR) ranges from 1 to themaximum number of auctioneers (MaxAucs) and maximum number of bidders (MaxBdrs), respectively. Hence, there are two parameters in the model: MaxAucs (line 2) and MaxBdrs (line 7). 
Listing 1. Declarations for the CNP-ext CPN model

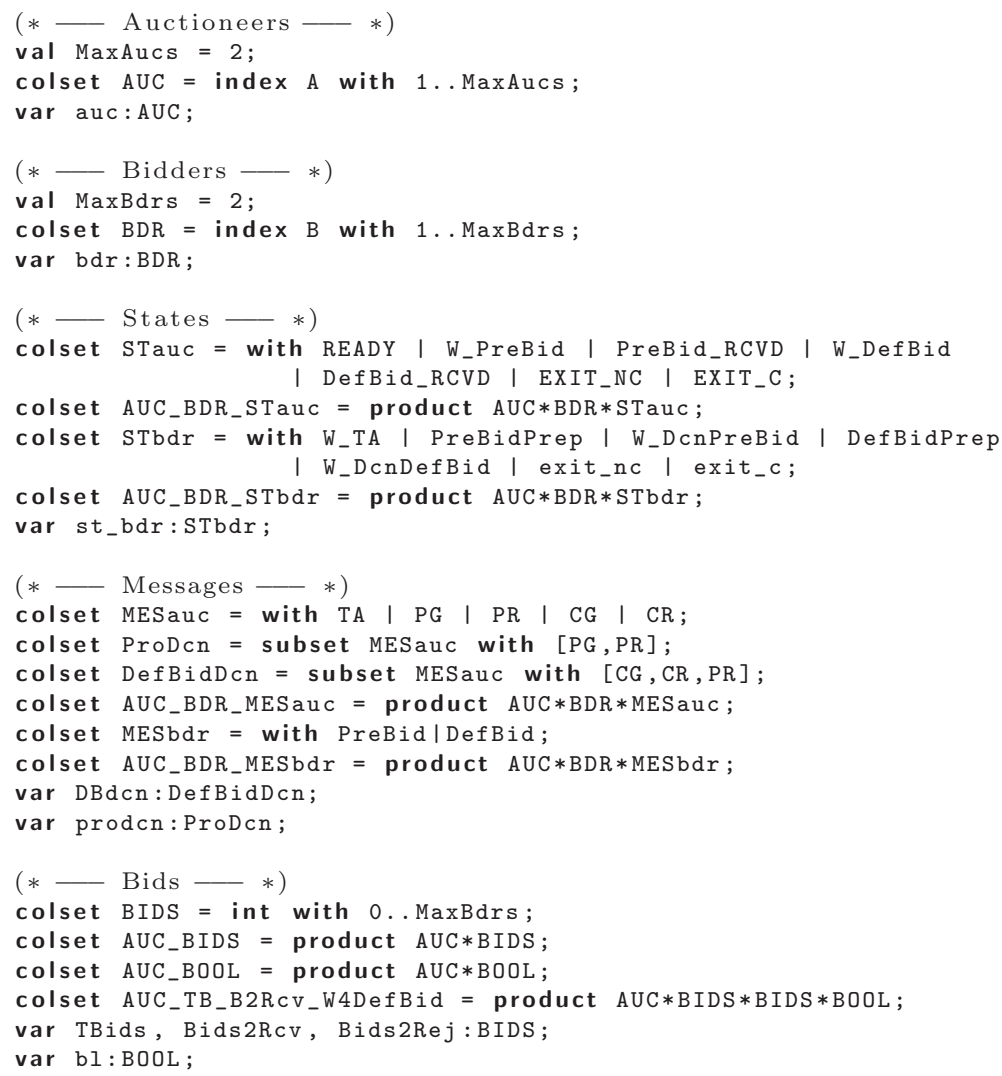

The states of the auctioneers and bidders are defined by the colour sets STauc (lines 12-13) and STbdr (lines 15-16) respectively, as defined in Table 1. The colour sets MESauc (line 21) and MESbdr (line 25) define the messages sent from auctioneers to bidders (Task Announcement-TA, Provisional Grant-PG, Provisional Reject-PR, Confirm Grant-CG, Confirm Reject-CR) and bidders to auctioneers (PreBid, DefBid) respectively. In the model we encode multiple auctioneers and bidders within the data structures, rather than in the net structure, thus allowing the number of auctioneers and bidders to be changed without requiring changes to the net structure. Accordingly, we associate the auctioneer's states and messages with the identity of an auctioneer and a bidder in the colour sets AUC_BDR_STauc (line 14) and AUC_BDR_MESauc (line 24), respectively. It is a similar situation for the bidders, thus the colour sets AUC_BDR_STbdr (line 17) and AUC_BDR_MESbdr (line 26) associate the identity of an auctioneer and bidder with the states and messages of a bidder, respectively. The colour set AUC_TB_B2Rcv_W4DefBid (line 34) defines a 4-tuple. It records the identity of the auctioneer, the total number of bidders participating, the number of bids to be received, and if the auctioneer is waiting for a Definitive Bid. 


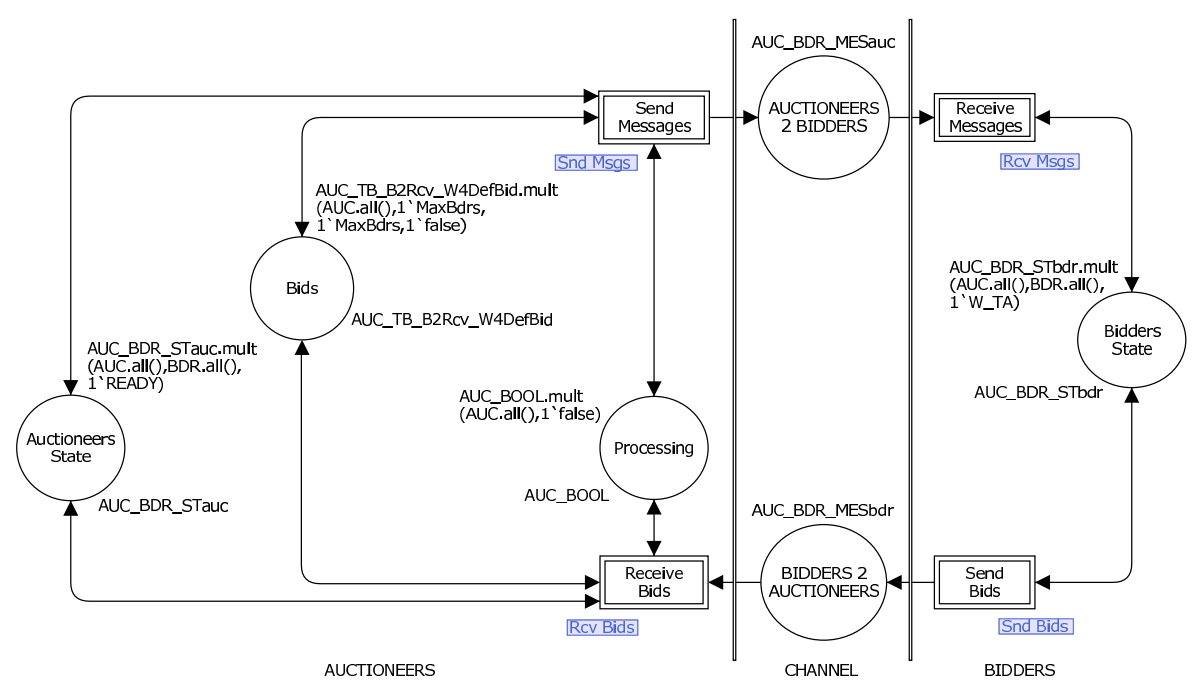

Fig. 3. Main Page of the CPN Model

\subsection{Model Structure}

The top-level page of the hierarchically constructed CNP-ext CPN model is shown in Fig. 3. It contains 6 places and 4 substitution transitions (double-rectangles, each representing another model page) and provides the main structure for the protocol. The auctioneers are modelled on the left and the bidders on the right. They communicate via two places shown in the middle of the figure, AUCTIONEERS 2 BIDDERS and BIDDERS 2 AUCTIONEERS, that represent a non-lossy but reordering channel for each direction of communication. Typed by the colour sets AUC_BDR_MESauc and AUC_BDR_MESbdr, at any instant, these places may contain the messages of the auctioneers and bidders, each coupled with the identity of an auctioneer and bidder. These two places are initially empty.

The place Auctioneers State is typed by the colour set AUC_BDR_STauc and stores the states of all the auctioneers with respect to all the bidders. It is initially marked by triples consisting of the cartesian product of all auctioneers with all bidders, paired with the READY state. For example for MaxAucs=2 and MaxB$\mathrm{drs}=2$, the initial marking would be $1^{\circ}(\mathrm{A}(1), \mathrm{B}(1), \mathrm{READY})++1^{\prime}(\mathrm{A}(1), \mathrm{B}(2)$, $\mathrm{READY})++1^{\circ}(\mathrm{A}(2), \mathrm{B}(1), \mathrm{READY})++1^{\circ}(\mathrm{A}(2), \mathrm{B}(2), \mathrm{READY})$, where ++ is multiset addition. The place Bidders State that models the states of the bidders can be described in a similar way.

The place Processing, typed by the colour set AUC_BOOL (see Listing. 1), keeps track of the auctioneers that are currently processing bids. In the initial marking, no auctioneers are processing bids.

The place Bids is typed by the colour set AUC_TB_B2Rcv_W4DefBid. It is initially marked with each of the auctioneers negotiating with MaxBdrs bidders, with MaxBdrs bids to be received, with none waiting for a Definitive Bid. 


\subsection{Model of the Auctioneers}

The behaviour of the auctioneers is modelled by the two substitution transitions, Send Messages and Receive Bids, in Fig. 3 .

Send Messages. This substitution transition represents the page shown in Fig. 4, which details the procedures for the auctioneers to send messages to the bidders. It consists of 6 executable transitions and 2 additional places. The place Bid Selected, typed by the colour set AUC_BOOL (see Listing 1), shows whether the auctioneers have selected a Definitive Bid in the negotiation process. The initial marking is such that none of the auctioneers have confirmed the grant of a Definitive Bid. The place Bids2Reject, typed by the colour set AUC_BIDS (see Listing 10, records the number of updated Pre Bids to reject after a Definitive Bid has been granted. The initial marking shows that no auctioneers have any bids to reject.

This page operates as follows. Initially, all the auctioneers are in the state READY with respect to all the bidders, and hence are ready to broadcast a Task Announcement to all the bidders. The Broadcasting TAs transition models an auctioneer initiating negotiations by sending a Task Announcement to each of the bidders. Firing this transition causes the selected auctioneer to change state to W_PreBid with respect to all the bidders, and a Task Announcement to be broadcast to each of the bidders. This transition is concurrently enabled for all auctioneers.

At some point, all bidders will respond to the TA with a Pre Bid. When the auctioneer has received all Pre Bids, it would be in the state PreBid_RCVD with respect to all the bidders, the marking of place Bids would indicate that no more bids need to be received, and a true value with respect to this auctioneer on place Processing indicates that the auctioneer can now process the bids. At this instant, the transition Snd Prov Dons (Send Provisional Decisions) would be enabled. When it fires it sends a provisional decision to each of the bidders. When there is only one bidder, it is sent either a Provisional Grant (PG) or a Provisional Reject (PR), modelled as a non-deterministic choice by the variable proden (see Listing 10. Accordingly, the auctioneer changes state to W_DefBid or W_PreBid respectively. When there is more than one bidder (MaxBdrs > 1), then one bidder is sent a PG (also modelled as a non-deterministic choice) and the rest are sent a PR. As before, the auctioneer changes state to W_DefBid with respect to the bidder that it sent the PG, and W_PreBid with respect to rest of the bidders. In either case, a false is returned to the place Processing with respect to this auctioneer, indicating that processing has finished for now.

Later, when the auctioneer receives the Definitive Bid (from the provisionally granted bidder) and all updated Pre Bids (from the provisionally rejected bidders), the transition Snd DefBid Dcn (Send Definitive Bid Decision) becomes enabled. This transition models the auctioneer's decision on the Definitive Bid, 


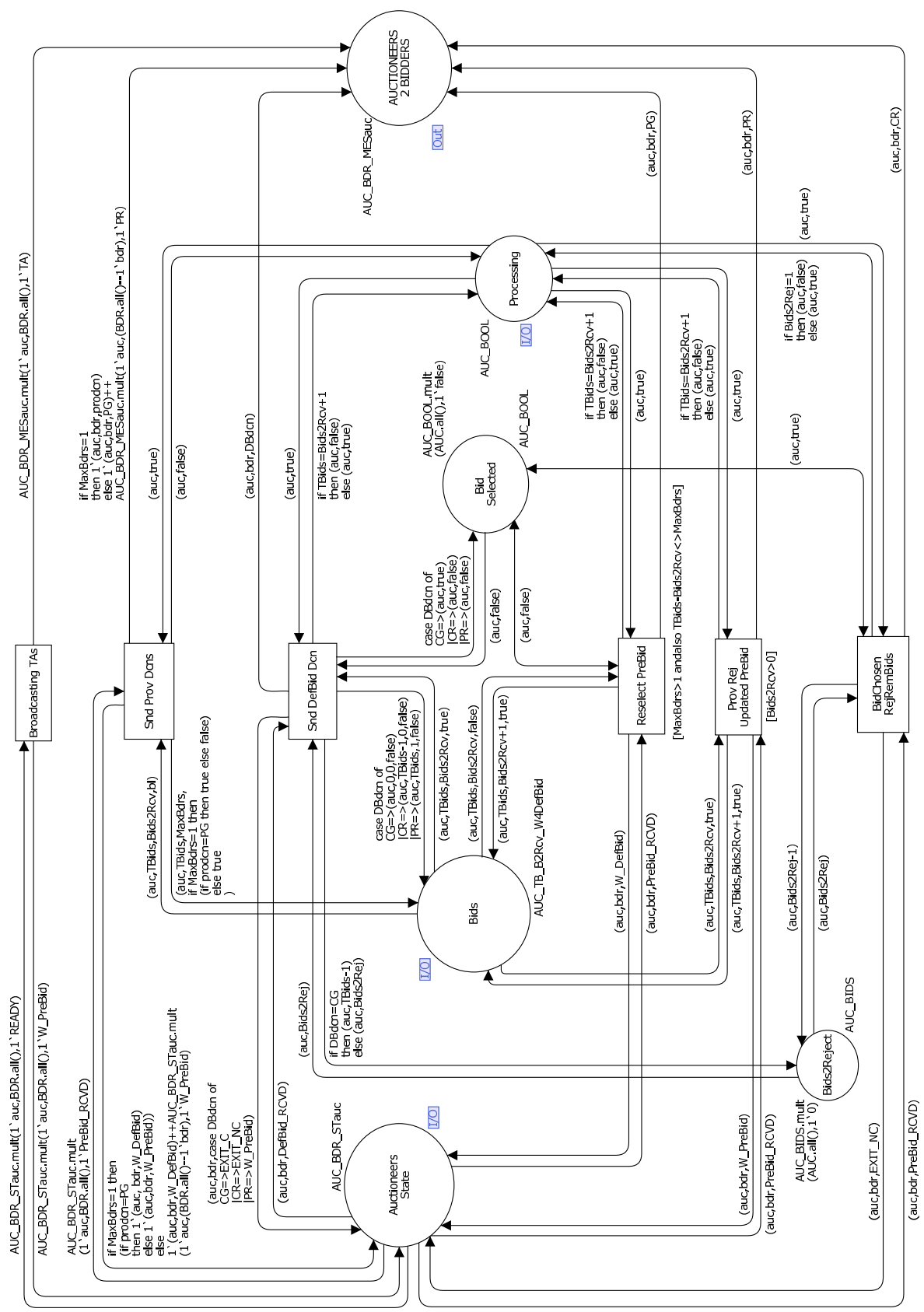

Fig. 4. Send Messages 
whether to send a Confirm Grant (CG), Confirm Reject (CR) or Provisional Reject (PR) to the corresponding bidder. In the case of CG, all other bidders are sent a $\mathrm{CR}$ and negotiations cease. In the case of either a CR or PR, another Pre Bid is selected, a PG is sent to the corresponding bidder, and the remaining bidders are sent a PR.

The outcome of the Definitive Bid decision is again non-deterministic, modelled by the variable DBden (see Listing 1). When the transition Snd DefBid Den fires, the auctioneer changes state to EXIT_C, EXIT_NC or W_PreBid depending upon the value of the variable DBdcn (either CG, CR or PR) as can be seen by the expression on the arc joining the transition to the place Auctioneers State. A CG decision deposits a true on the place Bid Selected while a CR or a PR retains the value to false. Also, the number of bidders to be sent a CR (recorded in the Bids2Reject place) is updated accordingly. The expression on the arc joining the transition to the place Bids updates the total bids and the number of bids to be received. In case of a CG decision, both become 0 as the auctioneer would have confirmed a Definitive Bid and hence would not be expecting any more bids. In case of a CR decision, the total bids would be decremented by 1 as the negotiation would with that bidder would cease, and the number of bids to be received would remain 0. Finally, in case of a Provisional Reject (PR) decision, the total bids would remain unchanged as the bidder would still be involved in the negotiation, and the number of bids to be received would be 1 . The condition on the arc joining the transition to the place Processing updates the processing status of this auctioneer accordingly.

If the auctioneer sends a CG to the bidder of the Definitive Bid, it then needs to send a CR to the remaining bidders. For this scenario, the transition BidChosen RejRemBids (Bid Chosen Reject Remaining Bids) is enabled, which sends a CR to all the remaining bidders. Its occurrence also causes the auctioneer to change state from PreBid_RCVD to EXIT_NC with respect to each of the rejected bidders. The expression on the arc joining the transition to the place Processing ensures that when the last bid is sent a CR (Bids2Rej=1), the auctioneer is no longer in the processing state.

If the auctioneer sends a CR or a PR to the bidder of the Definitive Bid, then the auctioneer needs to first reselect a Pre Bid (send a PG), and then provisionally reject all the remaining updated Pre Bids. These activities need to occur in sequence and are modelled by the transitions Reselect PreBid and Prov Rej Updated PreBid, where the transition Prov Rej Updated PreBid will only occur after the occurrence of Reselect PreBid.

The firing of Reselect PreBid causes the auctioneer to change state from PreBid_RCVD to W_DefBid and also sends a PG to the bidder. The boolean false is on the place Bid Selected with respect to this auctioneer, indicating that a Definitive Bid has not been selected. Also, the expression on the arc joining the transition to the place Bids increments the value of the bids to be received by 1 $($ Bids2Rcv +1$)$, and sets the auctioneer to be waiting for a Definitive Bid (shown by the boolean true). 


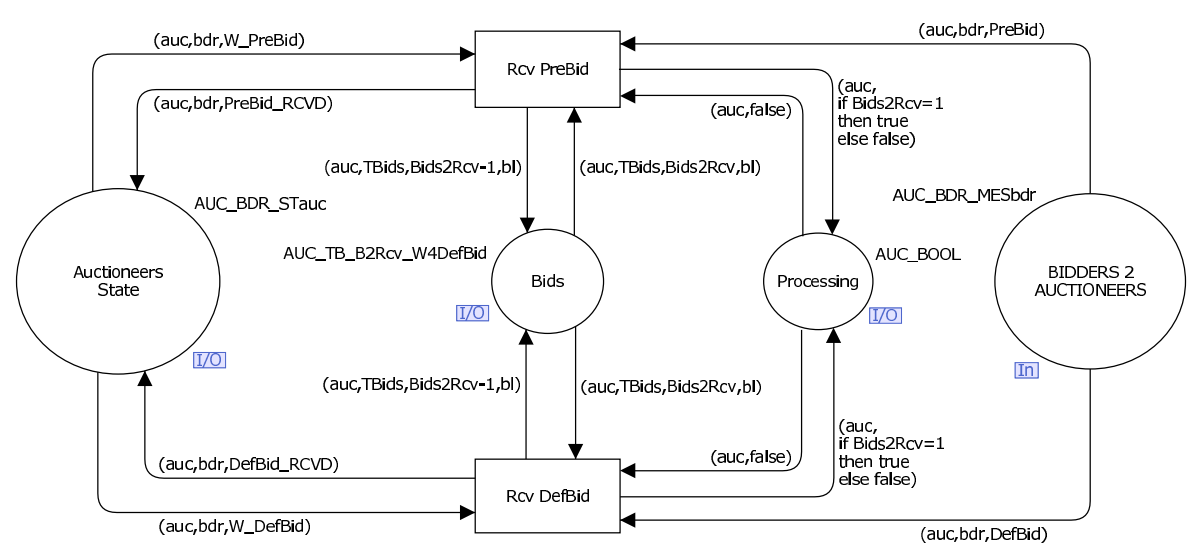

Fig. 5. Receive Bids

The transition Prov Rej Updated PreBid is used to reject the remaining updated Pre Bids only after the process of reselecting a Pre Bid has occurred, which is enforced by its guard. When it occurs, the auctioneer changes state from PreBid_RCVD to W_PreBid with respect to one bidder, and sends a PR message to that bidder. Each time the transition occurs, the number of bids to receive is incremented by 1 (the arc from Prov Rej Updated PreBid to the place Bids). When the auctioneer has finished sending a PR to all the remaining bidders, a boolean false is deposited on the place Processing as evaluated by the expression on the arc joining the transition to the place Processing.

Receive Bids. This substitution transition (see Fig. 5) models the reception of bids and comprises 2 executable transitions: Rcv PreBid (Receive Pre Bid), and Rcv DefBid (Receive Definitive Bid). The firing of Rcv PreBid removes a Pre Bid from the BIDDERS 2 AUCTIONEERS place, causes the auctioneer to change state to PreBid_RCVD with respect to the corresponding bidder, and decrements the number of bids to be received by one (arc from Rcv PreBid to Bids). The Rcv DefBid transition operates in exactly the same way, except that it receives a Definitive Bid from the channel and changes the state of the auctioneer to DefBid_RCVD with respect to the corresponding bidder. When the final bid is received, the processing status of the auctioneer is switched to true (arcs from the transitions to Processing) and the auctioneer can begin to process the bids and send responses.

\subsection{Model of the Bidders}

The behaviour of the bidders is modelled by the two substitution transitions, Receive Messages and Send Bids, from Fig. 3 .

Receive Messages. The page corresponding to this substitution transition is shown in Fig. 6 and comprises five transitions that model the reception of 


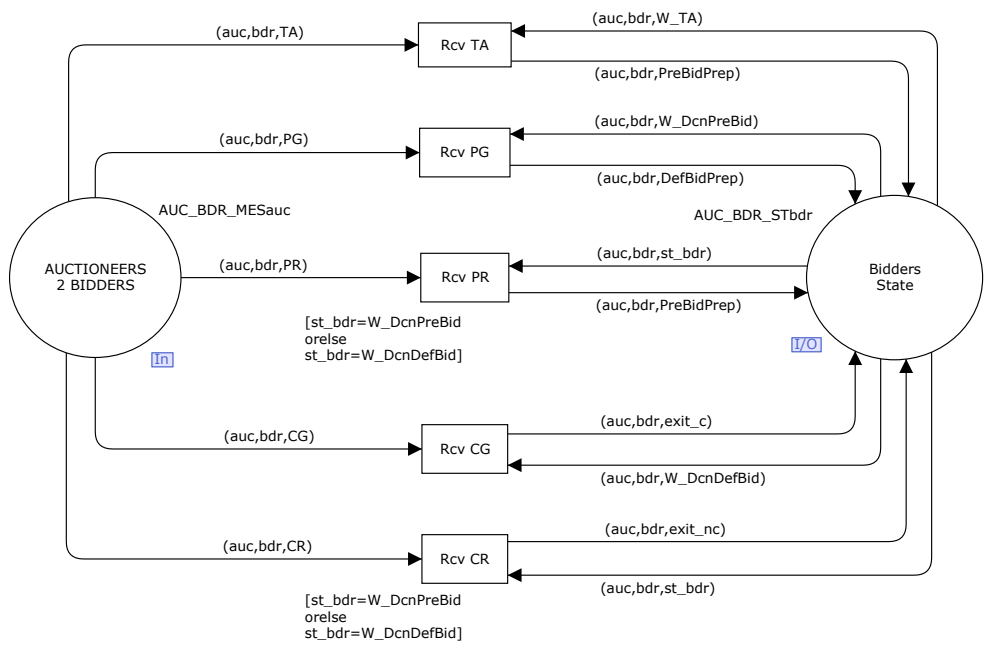

Fig. 6. Receive Messages

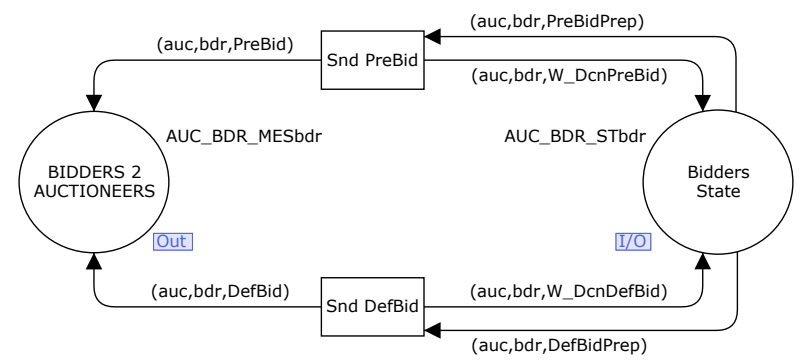

Fig. 7. Send Bids

messages from auctioneers by the bidders. Transition Rcv TA (Receive Task Announcement) models the reception of the Task Announcement, the firing of which causes the TA to be removed from the channel place and the state of the bidder to change from W_TA to PreBidPrep with respect to the corresponding auctioneer (seen in the inscriptions on the arcs between Rcv TA and the Bidders State place). Similarly, the firing of transitions Rcv PG (Receive Provisional Grant), Rcv CG (Receive Confirm Grant), Rcv PR (Receive Provisional Reject) and Rcv $\mathrm{CR}$ (Receive Confirm Reject) removes the respective message from the channel place and update the state of the bidder as shown in Fig. 6.

Send Bids. The page corresponding to this substitution transition is given in Fig. 7. It models the procedures for sending a Pre Bid (Snd PreBid) and a Definitive Bid (Snd DefBid). 


\section{State Space Analysis Results}

As all the auctioneers are independent entities and interact with the bidders in a similar way, we analyse the protocol properties for a single auctioneer. Table 2 shows the state space analysis results generated by CPN Tools for different values of MaxBdrs and Fig. 8 shows the reachability graph for one auctioneer and one bidder.

From Table 2, we see that the size of the state space increases as the number of bidders increases. Also, in each case, the number of Strongly Connected Components (Scc's) is less than that in the state space, signifying the presence of cyclic behaviour in the system. This is evident in Fig. 8 and is expected.

\subsection{Absence of Deadlocks and Consistency in Beliefs}

We can observe from Table 2 that in each case the number of dead markings is one more than MaxBdrs, i.e. No. of Dead Markings $=$ MaxBdrs +1 . This matches the results obtained in [3] for the Contract Net Protocol, which has a single auctioneer dealing with multiple bidders simultaneously. In each case analysed, one of the dead markings corresponds to no contract being established at the end of the negotiations (marking 14 in Fig. 8). This is caused by the auctioneer firmly rejecting each definitive bid, thus ending communication with the corresponding bidder, until no bidders remain. The other MaxBdr dead markings correspond to a contract being formed between the auctioneer and one of the MaxBdr bidders. For MaxBdrs=1, there exists only one such dead marking (marking 15 in Fig. 8). All these MaxBdrs+1 dead markings represent expected termination of the protocol. This is illustrated with the help of Fig. 9, which shows the node descriptors of the dead markings for the case of MaxBdrs $=3$ and $\operatorname{MaxAucs}=1$.

In each of the dead markings in Fig. 9, the place Bids contains a token, (A(1),0,0,false). This records the auctioneer's identity $(\mathrm{A}(1))$, that no bidders are still involved in negotiations (the first 0 ), that the auctioneer is not expecting any more bids (the second 0 ), and is not waiting for a definitive bid from a bidder (false). Additionally, all dead markings show that the Processing place

Table 2. State space analysis results as a function of the parameter MaxBdrs

\begin{tabular}{|c|c|c|c|c|c|}
\hline Properties/MaxBdrs & 1 & 2 & 3 & 4 & 5 \\
\hline State Space Nodes & 15 & 115 & 934 & 7761 & 63542 \\
State Space Arcs & 16 & 187 & 2101 & 22661 & 228841 \\
Time (hh:mm:ss) & 00:00:00 & $00: 00: 00$ & $00: 00: 01$ & $00: 00: 18$ & $00: 21: 15$ \\
Scc Graph Nodes & 7 & 50 & 290 & 1546 & 7658 \\
Scc Graph Arcs & 6 & 95 & 853 & 7153 & 59221 \\
Dead Markings & 2 & 3 & 4 & 5 & 6 \\
Home Space (Dead Markings) & true & true & true & true & true \\
Dead Transition Instances & 3 & 1 & none & none & none \\
Channel Bound & 1 & 2 & 3 & 4 & 5 \\
\hline
\end{tabular}




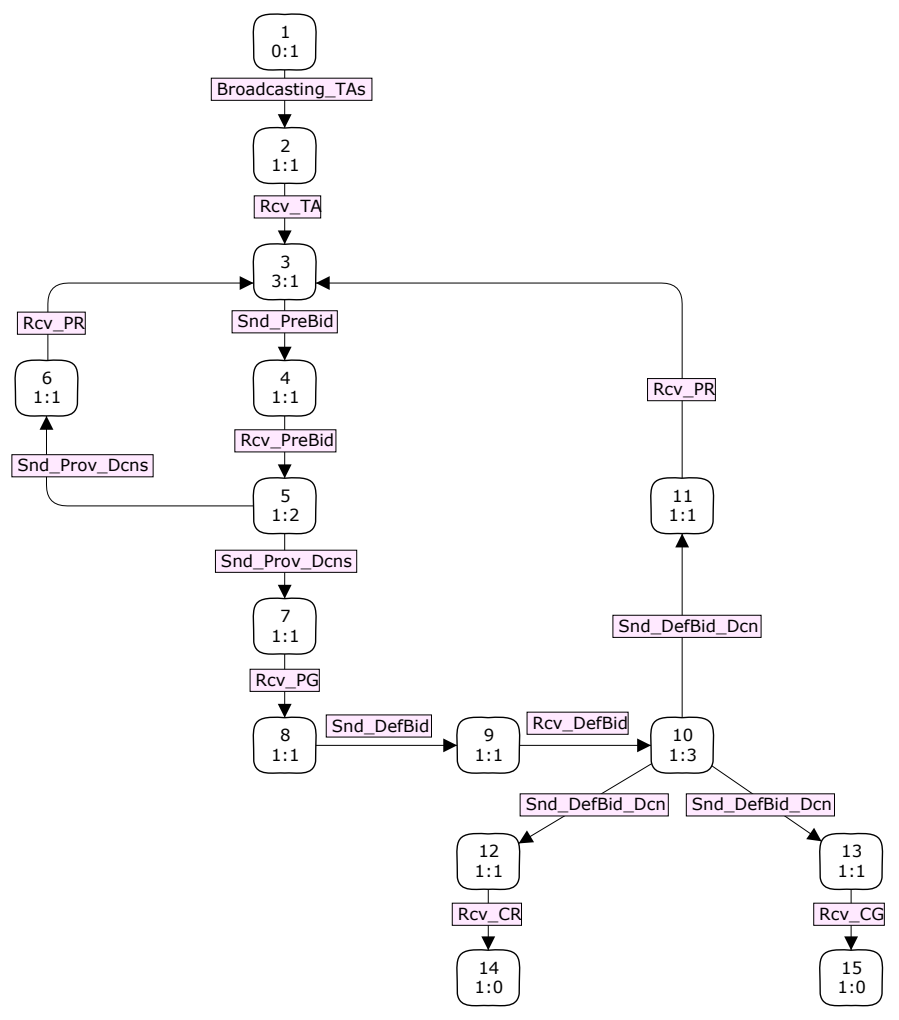

Fig. 8. Reachability Graph (MaxAucs = MaxBdrs = 1)

contains the token $(\mathrm{A}(1)$,false), meaning $\mathrm{A}(1)$ has replied to all bidders and is no longer processing bids, the Bids2Reject place contains the token $(A(1), 0)$, meaning there are no more bids left to be rejected, and both channel places are empty, meaning there are no unprocessed messages or bids. This all represents expected and desirable behaviour.

The markings differ, however, on the Auctioneers State, Bidders State and Bid Selected places. Marking 925 corresponds to the case where no contract is formed at the end of the negotiations. Hence, the marking of these places indicates that the auctioneer exited without a contract with any of the bidders, all bidders exited without a contract with the auctioneer, and no bid was selected, respectively.

The other three dead markings, 572, 547 and 522, correspond to the cases where a contract was awarded to bidder 1, 2 and 3 respectively. The marking of Bid Selected is true for A(1) in all three markings. The marking of Auctioneers State shows that the auctioneer exited with a contract with respect to one of the bidders, and no contract with respect to the other bidders. The marking of Bidders State shows that one bidder exited with a contract while the others did not. In all three markings, the auctioneer exited with a contract with respect to the bidder that exited with a contract. Hence, consistency of belief holds 


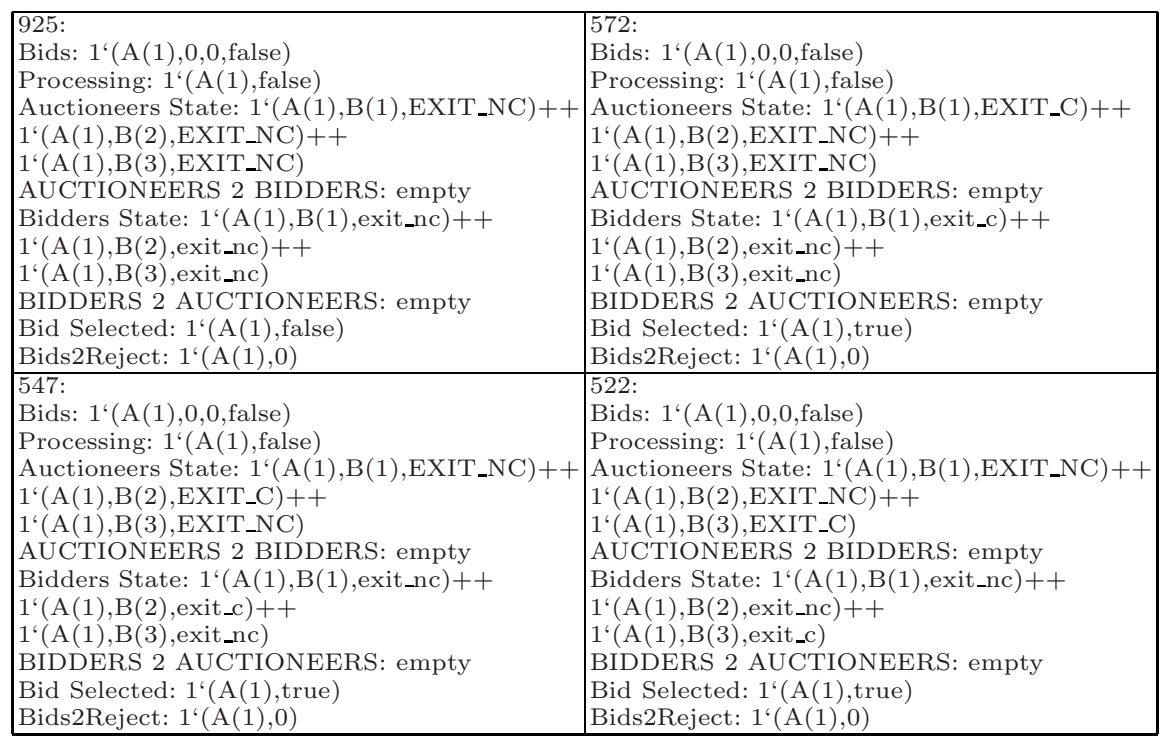

Fig. 9. Node Descriptors for the dead markings (MaxAucs $=1$ and MaxBdrs $=3$ )

for these three markings, as it does for marking 925. We conjecture that this property holds for any value of MaxBdrs.

\subsection{Absence of Livelocks and Proper Termination}

Although the system exhibits cyclic behaviour, it does not livelock. This is shown in Table 2, where we record the result of a state space query that checks whether all dead markings form a home space. A home space is a set of markings with the property that all markings can reach at least one of its members. The results of the query show that all markings can reach at least one dead marking, given suitable fairness assumptions, hence there are no livelocks. We conjecture that this property holds for any value of MaxBdrs. Because the system can always reach at least one dead marking, and that all dead markings are desirable, we conclude that the system terminates correctly.

\subsection{Absence of Dead Code}

Dead transitions equate to dead code. From Table 2 for $3 \leq$ MaxBdrs $\leq 5$ we see that there are no dead transitions. We conjecture that this holds for all MaxBdrs $\geq 3$. However, for MaxBdrs $=1$ we see that there are three dead transitions. These correspond to the transitions Reselect PreBid, Prov Rej Updated PreBid and BidChosen RejRemBids. An auctioneer may reply to a Definitive Bid with either a $\mathrm{CG}, \mathrm{CR}$ or a $\mathrm{PR}$. If the auctioneer replies with a $\mathrm{CG}$, then there are no other bidders to which CR's need be sent, hence BidChosen RejRemBids is 
dead. If the auctioneer replies with a $\mathrm{CR}$ or PR, there are no Pre Bids for the auctioneer to provisionally grant, hence no other Pre Bids to provisionally reject. Hence Reselect PreBid and Prov Rej Updated PreBid are dead. Therefore, this result is expected. We also see from Table 2 that there is one dead transition when MaxBdrs=2. This corresponds to the transition Prov Rej Updated PreBid. If there are only two bidders, and the auctioneer responds with a CR or PR to a Definitive Bid from one of them, the only Pre Bid is provisionally granted and hence there are no other Pre Bids for the auctioneer to provisionally reject. This is also expected behaviour.

\subsection{Channel Bound}

Table 2 shows that the channel places are bounded by MaxBdrs for all cases we examined. This can be explained by noting that the single auctioneer interacting with MaxBdrs bidders will, at any instant, send no more than a single message to each of the MaxBdrs bidders, hence a bound of MaxBdrs messages. Similarly, each bidder, at any instant, sends a single message to the auctioneer in reply, hence a bound of MaxBdrs bids.

\section{Conclusions and Future Work}

In this paper, we have presented, for the first time, an abstract parametric model of the CNP-ext [1] and analysed the protocol using the state space techniques. Our model captures the multithreaded nature of the auctioneers dealing with the bidders concurrently and provides a semantics for the Protocol Flow Diagram representation in [13]. We have proved a number of properties for one auctioneer and a number of bidders from 1 to 5 . We have shown that the protocol will always terminate correctly, and that there is consistent belief between the auctioneer and bidders. We have demonstrated that there are no livelocks, and that the only dead transitions are expected. Finally, we have also shown that both the channel bounds are limited to MaxBdrs. We conjecture that these properties hold for all MaxBdrs $>0$.

In the future we would like to extend the verification of CNP-ext to any number of bidders, not just 1 to 5. We would then like to relax Assumptions 1 and 4, by extending the model to open multi-agent systems and introducing deadlines, respectively. Finally, we would like to extend this work to the Extended Contract Net Protocol [5]6] and the Provisional Agreement Protocol [14]15/16/17/18 which are more elaborate and complex than CNP-ext.

\section{References}

1. Aknine, S., Pinson, S., Shakun, M.F.: An Extended Multi-Agent Negotiation Protocol. Autonomous Agents and Multi-Agent Systems 8(1), 5-45 (2004)

2. Billington, J., Gallasch, G.E., Han, B.: A Coloured Petri Net Approach to Protocol Verification. In: Desel, J., Reisig, W., Rozenberg, G. (eds.) Lectures on Concurrency and Petri Nets 2003. LNCS, vol. 3098, pp. 210-290. Springer, Heidelberg (2004) 
3. Billington, J., Gupta, A.K.: Effectiveness of Coloured Petri nets for Modelling and Analysing the Contract Net Protocol. In: Proceedings of 8th Workshop and Tutorial on Practical Use of Coloured Petri Nets and the CPN Tools, Aarhus, Denmark, October 22-24, 2007, pp. 49-65 (2007)

4. Ferber, J.: Multi-Agent Systems: An Introduction to Distributed Artificial Intelligence. Addison-Wesley Longman, Amsterdam (1999)

5. Fischer, K., Kuhn, N.: A DAI Approach to Modelling the Transportation Domain, DFKI Research Report RR-93-25. German Research Centre for Artificial Intelligence (DFKI), Saarbrücken (1993)

6. Fischer, K., Müller, J.P., Heimig, I., Scheer, A.W.: Intelligent Agents in Virtual Enterprises. In: Proceedings of the 1st International Conference on the Practical Application of Intelligent Agents and Multi-Agent Technology, London, UK, pp. 205-223 (1996)

7. Foundation for Intelligent Physical Agents (FIPA), http://www.fipa.org/specs/ fipa00029/SC00029

8. ISO/IEC. Software and Systems Engineering - High-level Petri Nets - Part 1: Concepts, Definitions and Graphical Notation. ISO/IEC 15909-1, 1 (December 2004)

9. Jensen, K.: Coloured Petri Nets: Basic Concepts, Analysis Methods and Practical Use, 2nd edn. Monographs in Theoretical Computer Science, vol. 1 to 3. Springer, Heidelberg (1997)

10. Jensen, K., Kristensen, L.M., Wells, L.: Coloured Petri Nets and CPN Tools for Modelling and Validation of Concurrent Systems. International Journal on Software Tools for Technology Transfer 9(3-4), 213-254 (2007)

11. Paurobally, S.: Rational Agents and the Processes and States of Negotiation. PhD thesis, Imperial College, London, UK (2002)

12. Paurobally, S., Cunningham, J., Jennings, N.R.: Verifying the Contract Net Protocol: A Case Study in Interaction Protocol and Agent Communication Language Semantics. In: Proceedings of 2nd International Workshop on Logic and Communication in Multi-Agent Systems, Nancy, France, pp. 98-117 (2004)

13. Perugini, D.: Agents for Logistics: A Provisional Agreement Approach. PhD thesis, The University of Melbourne, Victoria, Australia (2006)

14. Perugini, D., Lambert, D.: A Distributed Agent Approach to Global Transportation Scheduling. In: Proceedings of IEEE/WIC International Conference on Intelligent Agent Technology (IAT), Halifax, Canada (2003)

15. Perugini, D., Lambert, D.: Distributed Information Fusion Agents. In: Proceedings of the 6th International Conference on Information Fusion, Cairns, Australia (2003)

16. Perugini, D., Lambert, D.: Agent-Based Transport Scheduling in Military Logistics. In: Kudenko, D., Kazakov, D., Alonso, E. (eds.) AAMAS 2004. LNCS (LNAI), vol. 3394. Springer, Heidelberg (2004)

17. Perugini, D., Lambert, D.: Provisional Agreement Protocol for Global Transportation Scheduling. In: Proceedings of International Workshop Agent in Traffic and Transportation as part of AAMAS 2004, New York, U.S (2004)

18. Perugini, D., Lambert, D.: From Single Static to Multiple Dynamic Combinatorial Auctions. In: Proceedings of IEEE/WIC International Conference on Intelligent Agent Technology (IAT), Compiegne University of Technology, France (2005)

19. Smith, R.G.: The Contract Net Protocol: High-Level Communication and Control in a Distributed Problem Solver. IEEE Transactions On Computers C-29(12), 1104-1113 (1980) 